\title{
Inverse methods for characterizing the anisotropic hyperelastic behaviour of arteries in vitro
}

\author{
S. Avril ${ }^{1, a}$, P. Badel ${ }^{1}$, and K. Genovese ${ }^{2}$ \\ 1 Center for Health Engineering, Ecole des Mines de Saint-Etienne, CNRS UMR 5146, IN- \\ SERM IFR 143, 158 Cours Fauriel, 42023 SAINT-Etienne cedex 2, FRANCE \\ 2 Università degli Studi della Basilicata, Viale dell'Ateneo Lucano 10, 85100, Potenza, ITALY
}

\begin{abstract}
In this paper, we present a bi-axial characterization of in vitro human arteries. From the full-field experimental data obtained in inflation/extension tests, an inverse approach, called the virtual fields method (VFM), is used for deriving the material parameters of the tested arterial segment. The obtained results are promising and the approach can effectively provide relevant values for the anisotropic hyperelastic properties of the tested sample.
\end{abstract}

\section{Introduction}

The combination of 3-D deformation measurements [1-7] and inverse approaches [8] is now very common in solid mechanics but it is still under-employed for identifying the anisotropic hyperelastic properties of the arterial tissues [9,10]. Moreover, the Virtual Fields Method [11], which is an inverse method specifically dedicated to full-field data, has never been used for the mechanical identification of arterial tissues although it has very relevant assets: insensitivity to the uncertainty of boundary conditions [11], robustness [12], fast convergence [13].

This paper attempts to give a new contribution for addressing the mechanical identification of arterial tissues by presenting an implementation of the Virtual Fields Method to full-field experimental data measured on the whole surface of an arterial segment during inflation/extension tests. The objective of the paper is to present the principle of the approach and to prove its feasibility on an example.

\section{Materials and Methods}

\subsection{Materials}

Results reported in this paper are obtained on a fairly straight segment of a human ascending aorta (initial length: $L_{0} \simeq 35 \mathrm{~mm}$, initial average radius: $R_{0} \simeq 10 \mathrm{~mm}$, initial average thickness: $e_{0} \simeq 1.3 \mathrm{~mm}$ ). This vascular segment has been obtained from a cadaveric 65 years old female donor. All procedures were carried out in accordance with the guidelines of the Institutional Review Board of the University Hospital Centre of Saint-Etienne, France. After gently cleaning the artery with physiological solution, two side branches were clamped with surgical suture threads (Fig. 1).

\footnotetext{
a e-mail: avril@emse.fr
} 

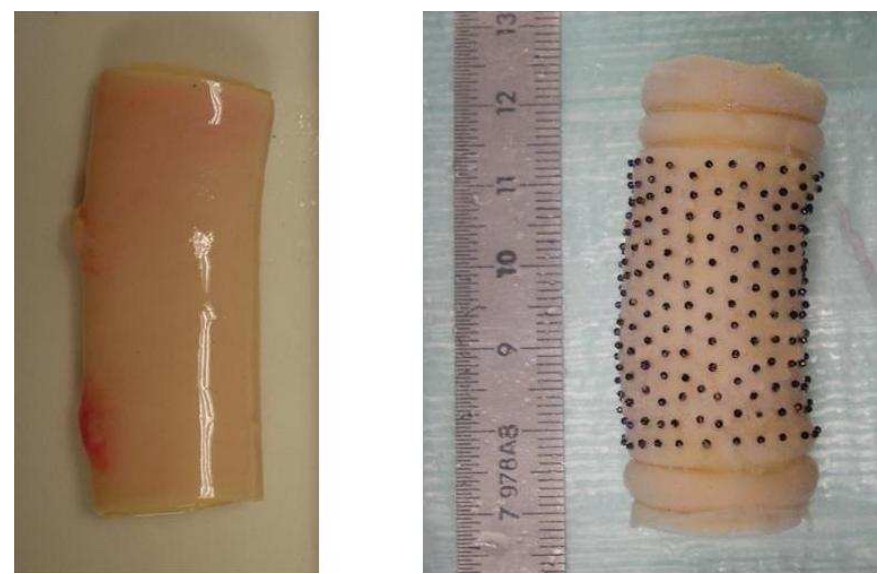

Fig. 1. Picture of the arterial segment used in the tests.

\subsection{Experiments}

The entire experimental setup used in this study was presented in [6]. The sample was mounted on the in vitro rig and preconditioned via 8 pressurization cycles from $p=0$ to $p=80 \mathrm{mmHg}$ $(10.5 \mathrm{kPa})$ and then 8 cycles of axial stretching from $L / L_{0}=1$ to $L / L_{0}=1.4$. Then, different sets of inflation/extension tests were performed.

Let $\boldsymbol{\Psi}_{t}$ denote the geometrical deformation of the artery induced by the loading at time $t$, such as $\mathbf{x}(t)=\boldsymbol{\Psi}_{t}\left(\mathbf{x}_{0}\right)$, where $\mathbf{x}(t)$ is the position vector of a material point at $t$ and $\mathbf{x}_{0}$ is the initial position vector of the same material point.

The vascular segment is covered with $N(N \simeq 300)$ black spherical markers (Fig. 1). The optical measurement method was presented in [6]. It provides a point-wise evaluation of $\boldsymbol{\Psi}_{t}$ at all the points where a marker has been bonded. Accuracy of the measurement in terms of displacement magnitude is estimated to $0.17 \mathrm{~mm}[6]$.

Then the surface of the artery is meshed for interpolating $\boldsymbol{\Psi}_{t}$ across the whole artery from the data measured at the marker position. Triangular elements with linear interpolation functions are used. The mesh is defined using a Delaunay triangularization algorithm applied onto the marker initial positions (actually the marker position means the contact position of the marker onto the surface of the artery). Each triangular element is labeled by an index denoted $q$. Let $\mathbf{x}^{q}(t)$ denote the position vector of the center of gravity of each element at time $t$

Eventually, $\boldsymbol{\Psi}_{t}$ is known everywhere across the meshed area of the artery. Let $\Omega$ be this domain across which the deformation is measured. $\Omega$ is defined in the initial configuration because the deformation is Lagrangian. Let $\omega(t)$ be the counterpart of $\Omega$ in the deformed configuration at time $t$.

\subsection{Derivation of the field of Green-Lagrange strain tensors}

All the derivations presented in this paper are based on the theory of finite deformations [14]. Let $\mathbf{F}\left(\mathbf{x}_{0}, t\right)$ denote the deformation gradient: 


$$
\mathbf{F}\left(\mathbf{x}_{0}, t\right) \equiv \nabla \Psi_{t}\left(\mathbf{x}_{0}\right)
$$

where $\boldsymbol{\nabla}$ is the gradient operator.

Then we define the right Cauchy-Green tensor:

$$
\mathbf{C}\left(\mathbf{x}_{0}, t\right) \equiv \mathbf{F}^{T} \mathbf{F}
$$

where $\mathbf{F}^{T}$ is the transpose of $\mathbf{F}$.

Eventually, we define the Green-Lagrange strain tensor:

$$
\mathbf{E}\left(\mathbf{x}_{0}, t\right) \equiv[\mathbf{C}-\mathbf{I}] / 2
$$

where $\mathbf{I}$ is the identity tensor.

\subsection{Constitutive equations}

Arteries in vitro are usually considered as anisotropic visco-hyperelastic materials. Here, the viscoelastic properties are not considered and only the instantaneous and monotonic response is modeled, after preconditioning. Pseudo-elasticity can therefore be applied [15]. An anisotropic hyperelastic model is considered.

In this model, the Cauchy stress tensor, denoted $\mathbf{S}$, is deduced from the Green-Lagrange strain tensor such as:

$$
\mathbf{S}=\mathbf{F}: d \Phi / d \mathbf{E}: \mathbf{F}^{T}+\lambda \mathbf{I}
$$

where $\Phi$ is the strain energy function depending only on $\mathbf{E}$ and on material parameters. The term $\lambda \mathbf{I}$ comes from the incompressibility and $\lambda$ is assessed by writing the plane stress condition $\left(S_{33}=0\right)$.

Different strain energy functions for the anisotropic hyperelastic behavior of arteries may be found in the literature. Some of them are phenomenological $[15,16]$ and others are based on the microstructure [17]. Ignoring the microstructure, we chose the 2-D Fung model [15], which may be written as:

$$
\Phi=\frac{1}{2} \beta\left[\exp \left(\alpha_{11}\left(E_{11}\right)^{2}+\alpha_{22}\left(E_{22}\right)^{2}+\alpha_{12} E_{11} E_{22}\right)-1\right]=\frac{1}{2}[\beta \exp (Q)-1]
$$

where $\beta, \alpha_{11}, \alpha_{22}$ and $\alpha_{12}$ are material parameters and $E_{i j}$ are the components of the $\mathbf{E}$ matrix in the material coordinate system (indice 1 refers to the circumferential direction and indice 2 refers to the axial direction).

\subsection{Inverse approach}

The Virtual Fields Method (VFM) is an approach dedicated to the identification of material parameters from full-field measurements $[11-13,18]$. The principle may be summarized in two steps:

1. computing stress fields from Eq. 2 with the measured values of $E_{i j}$ and a guess for the material parameters,

2. finding the guess of material parameters that yields statically admissible stress fields.

Assuming quasi-static conditions and no body forces, step 2 may be written globally (weak form) such as:

$$
-\int_{\omega(t)} \mathbf{S}: \mathbf{E}^{*} d v+\int_{\partial \omega(t)} \mathbf{t} \cdot \mathbf{u}^{*} d s=0
$$

where $\partial \omega(t)$ is the boundary of the volume in the deformed configuration, vector $\mathbf{t}$ denotes the tractions applied onto this boundary (pressure forces and axial load), $\mathbf{u}^{*}$ is a displacement virtual field (i.e. a fake displacement field which acts as a test function in Eq. 3). $\mathbf{E}^{*}$ is the strain virtual field deduced from the gradient of $\mathbf{u}^{*}$ : 


$$
\mathbf{E}^{*}=\frac{1}{2}\left(\nabla \mathbf{u}^{*}+\nabla^{T} \mathbf{u}^{*}\right)
$$

The choice of virtual fields was largely discussed in the literature [11-13,18], but only when the VFM is applied in infinitesimal deformation conditions. No rule has been established for finite deformations. Empiric rules will be used here, yielding virtual fields $\mathbf{u}^{* 1}$ and $\mathbf{u}^{* 2}$. A constraint is that the virtual fields zero the action of unknown reaction forces or involve their resultant if the resultant is measured [11].

At position $\mathbf{x}$ where a marker is located, $\mathbf{u}^{* 1}$ and $\mathbf{u}^{* 2}$ take the following values:

$$
\left\{\begin{array}{l}
\mathbf{u}^{* 1}=\sin \left(\pi \frac{x_{3}-x_{3}^{\mathrm{b}}}{x_{3}^{\mathrm{t}}-x_{3}^{\mathrm{b}}}\right) \mathbf{i}^{\prime} \\
\mathbf{u}^{* 2}=\frac{x_{3}-x_{3}^{\mathrm{t}}}{x_{3}^{\mathrm{b}}-x_{3}^{\mathrm{t}}} \mathbf{k}^{\prime}
\end{array}\right.
$$

where vectors $\mathbf{i}^{\prime}$ and $\mathbf{k}^{\prime}$ are respectively the radial and longitudinal axes in the cylindrical coordinate system and $x_{i}(i=1,2,3)$ are the components of $\mathbf{x} ; x_{3}^{\mathrm{b}}$ stands for the value of $x_{3}$ at the bottom and $x_{3}^{\mathrm{t}}$ stands for the value of coordinate $x_{3}$ at the top; $x_{3}^{\mathrm{b}}$ and $x_{3}^{\mathrm{t}}$ are chosen such as all the positions satisfying $x_{3}^{\mathrm{b}} \leq x_{3} \leq x_{3}^{\mathrm{t}}$ lie in the $\omega(t)$ domain.

Eq. 5 only gives the values of the virtual displacement at marker positions. Between the marker positions, the values of $\mathbf{u}^{* 1}$ and $\mathbf{u}^{* 2}$ are interpolated using the linear interpolation scheme defined in section 2.2 .

Moreover, regarding the external virtual work, it can be written:

$$
\left\{\begin{array}{l}
\int_{\partial \omega(t)} \mathbf{t} \cdot \mathbf{u}^{* 1} d s=\int_{\partial \omega(t)} \mathbf{k}_{t} \cdot \mathbf{u}^{* 1} p(t) d s \\
\int_{\partial \omega(t)} \mathbf{t} \cdot \mathbf{u}^{* 2} d s=\int_{\partial \omega(t)} \mathbf{k}_{t} \cdot \mathbf{u}^{* 2} p(t) d s+\mathcal{F}(t)
\end{array}\right.
$$

where $\mathbf{k}_{t}$ is the outer normal vector at $t, \mathcal{F}(t)$ is the axial resultant load measured by a load cell. The contribution of this axial resultant load is not considered for $\mathbf{u}^{* 1}$ because: $\mathbf{u}^{* 1}\left(x_{3}^{\mathrm{b}}\right)=\mathbf{u}^{* 1}\left(x_{3}^{\mathrm{t}}\right)=\mathbf{0}$.

As $\mathbf{S}$ is deduced from experimental data, Eq. 3 cannot be satisfied exactly and the principle of the inverse method is to minimize the the squares of residuals, as defined in the following cost function:

$$
\zeta\left(\beta, \alpha_{11}, \alpha_{22}, \alpha_{12}\right)=\sum_{k} \sum_{m}\left[-\int_{\omega\left(t_{m}\right)} \mathbf{S}: \mathbf{E}^{* k} d v+p\left(t_{m}\right) \int_{\partial \omega\left(t_{m}\right)} \mathbf{k}_{t_{m}} \cdot \mathbf{u}^{* k} d s+\delta_{k 2} \mathcal{F}(t)\right]^{2}
$$

where $m$ labels the time when a measurement is achieved and $k$ labels the virtual field $(k=$ $1,2) ; \delta_{k 2}=0$ if $k=1$ and $\delta_{k 2}=1$ if $k=2$.

Considering the interpolation of all quantities with the triangular mesh, the previous integrals in the principle of virtual work may be changed into discrete sums.

$$
\begin{aligned}
& \zeta\left(\beta, \alpha_{11}, \alpha_{22}, \alpha_{12}\right)= \\
& \sum_{k} \sum_{m}\left[-\sum_{q} \mathbf{S}\left(\mathbf{x}^{q, m}\right): \mathbf{E}^{* k}\left(\mathbf{x}^{q, m}\right) A\left(\mathbf{x}^{q, m}\right) e\left(\mathbf{x}^{q, m}\right)+p\left(t_{m}\right) \sum_{q} \mathbf{k}_{t}\left(\mathbf{x}^{q, m}\right) \cdot \mathbf{u}^{* k}\left(\mathbf{x}^{q, m}\right) A\left(\mathbf{x}^{q, m}\right)+\delta_{k 2} \mathcal{F}(t)\right]^{2}
\end{aligned}
$$


where $A\left(\mathbf{x}^{q, m}\right)$ denotes the area of triangular element $q$ at time $t_{m}$ and $e\left(\mathbf{x}^{q, m}\right)$ denotes the thickness of the artery ${ }^{1}$ at $\mathbf{x}^{q}$ at time $t_{m}$.

Cost function $\zeta$ figures the quadratic gap between the internal virtual work (IVW) and the external virtual work (EVW) where:

$$
\begin{aligned}
& \mathrm{IVW}^{k, m}=e_{0} \sum_{q} \mathbf{S}\left(\mathbf{x}^{q, m}\right): \mathbf{E}^{* k}\left(\mathbf{x}^{q, m}\right) A\left(\mathbf{x}^{q, 0}\right) \\
& \mathrm{EVW}^{k, m}=p\left(t_{m}\right) \sum_{q} \mathbf{k}_{t}\left(\mathbf{x}^{q, m}\right) \cdot \mathbf{u}^{* k}\left(\mathbf{x}^{q, m}\right) A\left(\mathbf{x}^{q, m}\right)+\delta_{k 2} \mathcal{F}(t)
\end{aligned}
$$

Cost function $\zeta$ and IVW are driven by the choice of the unknown material parameters. Eventually, the cost function can be minimized through an iterative scheme using the NelderMead algorithm. This yields the unknown parameters $\beta, \alpha_{11}, \alpha_{22}$ and $\alpha_{12}$.

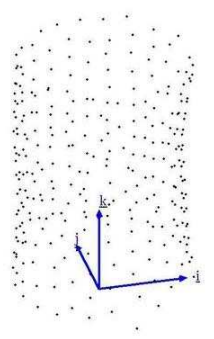

(a)

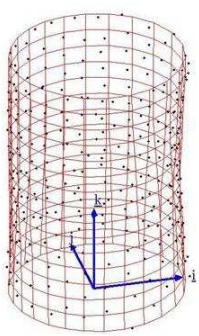

(b)

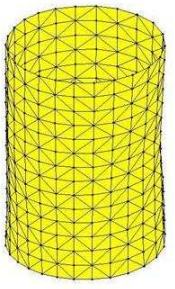

(c)

Fig. 2. Reconstruction and meshing of the initial geometry: (a) positions of the markers, (b) smooth surface fitting the positions of the markers and (c) mesh constructed on the smooth surface.

\section{Results}

A sample was tested for proving the feasibility of the approach (Fig. 1 and Fig. 2). We report data corresponding to pressure levels between 0 and $130 \mathrm{mmHg}$ at $L / L_{0}=1.1$ (Fig. 3).

The results obtained with the VFM are reported in Tab. 1. Convergence of the Nelder Mead optimization routine was reached in nearly 10 minutes. Actually, only three of the four parameters were identified by the VFM. Parameter $\alpha_{12}$ was set to 0.1 (value taken from [15]) because the optimization routine did not converge when $\alpha_{12}$ was among the unknowns.

\footnotetext{
${ }^{1}$ Due to the incompressibility assumption, $A\left(\mathbf{x}^{q, m}\right) e\left(\mathbf{x}^{q, m}\right)=A\left(\mathbf{x}^{q, 0}\right) e_{0}$, with $\mathbf{x}^{q, m}=\mathbf{x}^{q}\left(t_{m}\right)$.
} 

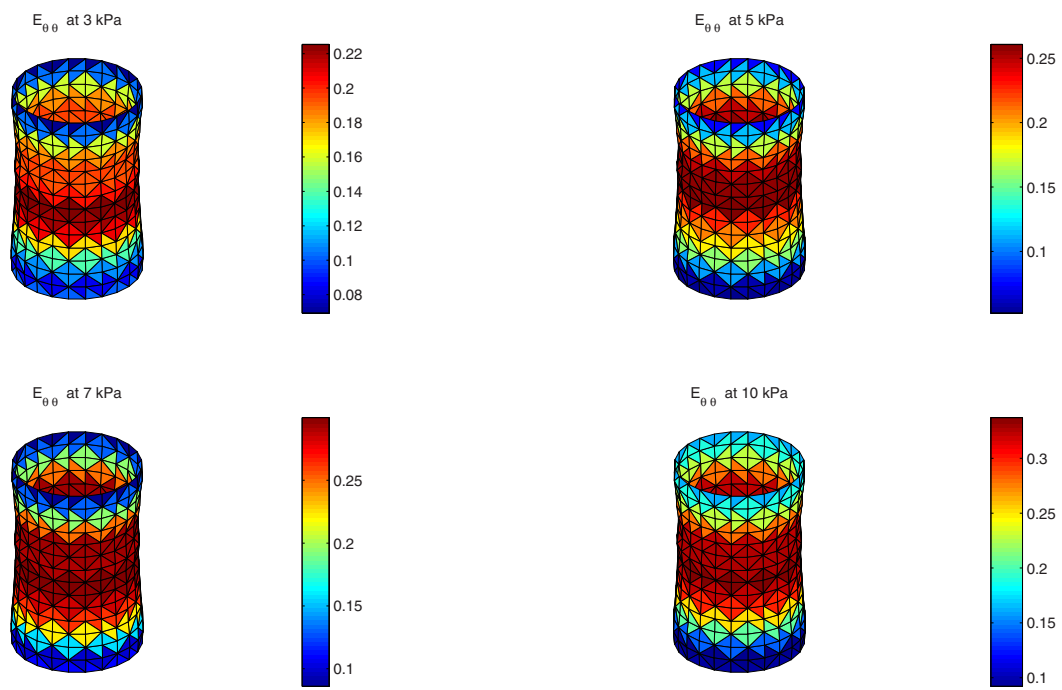

Fig. 3. Plot of the circumferential components of the Green-Lagrange strain tensor for different values of $p$.
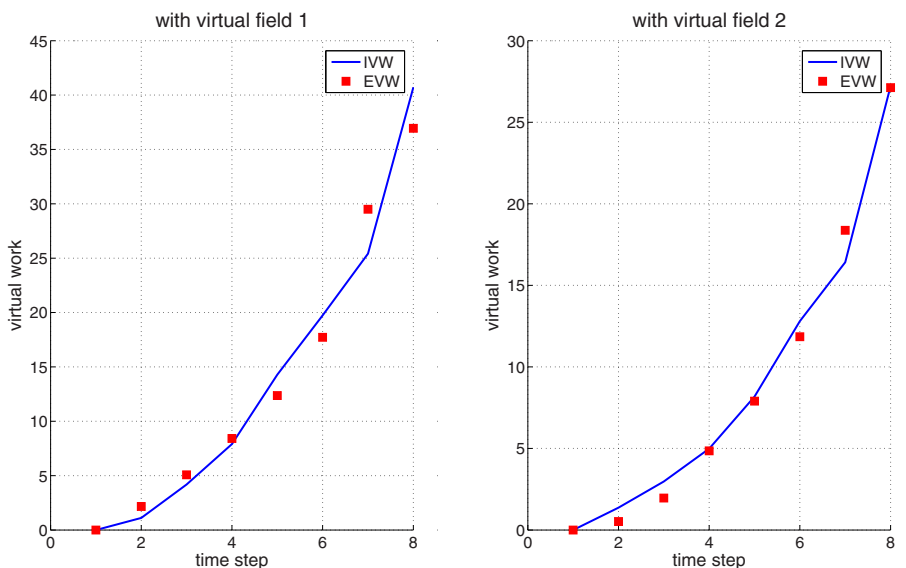

Fig. 4. Comparison of the internal and external virtual work for virtual field 1 (a) and virtual field 2 (b) when an anisotropic model is identitied

It can be observed in Fig. 4 that the gap between the external virtual work and the internal virtual work is very low for the obtained material parameters.

\section{Discussion}

The identified values for the material parameters of the artery are consistent with the range orders reported in the literature [15,17]. Parameter $\beta$ is usually around $10 \mathrm{kPa}$. It is interesting 


\begin{tabular}{|c|c|c|c|c|}
\hline parameter & $\beta$ & $\alpha_{11}$ & $\alpha_{22}$ & $\alpha_{12}$ \\
\hline identified value & $5 \mathrm{kPa}$ & 14.5 & 7 & 0.1 \\
\hline
\end{tabular}

Table 1. Results obtained with experimental data.

to notice the large difference between the value of $\alpha_{11}$ and $\alpha_{22}$. It means that there is a large anisotropy in this specimen, which has already been reported in the literature [17].

In [16], the implementation of the hyperelastic model of Eq. 2 in a FE code was discussed. In order to check the feasibility of utilizing this model with the parameters that we identified here, computations were achieved on the geometry of the artery with the Abaqus ${ }^{\circledR}$ software.

A comparison of the deformed geometry computed by Abaqus ${ }^{\circledR}$ to the measured deformed geometry is showed a very good agreement.

\section{Conclusions}

In conclusion, results presented in this work are promising regarding the application of the virtual fields method (VFM) for identifying the anisotropic hyperelastic properties of arteries. An innovative experimental device has been set up, calibrated and validated for characterizing arterial mechanical properties. It is now important to carry out a large number of experiments for validating the approach with different sets of data and different tests.

\section{Acknowledgements}

The authors would like to thank Professeur Jean-Pierre Favre and to his staff in the department of cardiovascular surgery at the University Hospital of Saint-Etienne (France), for their help in preparing the specimen tested in this study. This study is part of the Imandef project (Grant ANR-08-JCJC-0071) funded by the ANR (French National Research Agency).

\section{References}

1. P.K. Rastogi. Photomechanics. Springer Verlag, 1999.

2. C.G. Foster. Measurement of radial deformations in thin-walled cylinders. Experimental Mechanics, 18:426-430, 1978.

3. M.R. Viotti, A. Albertazzi, A.V. Fantin, and A. Dal Pont. Comparison between a whitelight interferometer and a tactile formtester for the measurement of long inner cylindrical surfaces. Optics and Lasers in Engineering, 46:396-403, 2008.

4. D.R. Matthys, J.A. Gilbert, and P. Greguss. Endoscopic measurement using radial metrology with digital correlation. Optical Engineering, 30(19):1400-1455, 1991.

5. K. Genovese. Radial metrology application to whole-body measurement on hyperelastic tubular samples. Optics and Lasers in Engineering, 45(11):1059-1066, 2007.

6. K. Genovese. A video-optical system for time-resolved whole-body measurement on vascular segments. Optics and Lasers in Engineering, 47:995-1008, 2009.

7. M.A. Sutton, X. Ke, S.M. Lessner, M. Goldbach, M. Yost, F. Zhao, and H.W. Schreier. Strain field measurements on mouse carotid arteries using microscopic three-dimensional digital image correlation. Journal of Biomedical Materials Research Part A, 84A(1):178190, 2007.

8. S. Avril, M. Bonnet, A.-S. Bretelle, M. Grédiac, F. Hild, P. Ienny, F. Latourte, D. Lemosse, S. Pagano, E. Pagnacco, and F. Pierron. Identification from measurements of mechanical fields. Experimental Mechanics, 48(5):381-402, 2008. 
9. P. Seshaiyer and J.D. Humphrey. A sub-domain inverse finite element characterization of hyperelastic membranes including soft tissues. Journal of Biomechanical Engineering, 125:363-371, 2003.

10. D.R. Einstein, A.D. Freed, N. Stander, B. Fata, and I. Vesely. Inverse parameter fitting of biological tissues: A response surface approach. Annals of Biomedical Engineering, 33(12):1819-1830, 2005.

11. M. Grédiac, F. Pierron, S. Avril, and E. Toussaint. The virtual fields method for extracting constitutive parameters from full-field measurements: a review. Strain, 42:233-253, 2006.

12. S. Avril, M. Grédiac, and F. Pierron. Sensitivity of the virtual fields method to noisy data. Computational Mechanics, 34(6):439-452, 2004.

13. S. Avril and F. Pierron. General framework for the identification of constitutive parameters from full-field measurements in linear elasticity. International Journal of Solids and Structures, 44:4978-5002, 2007.

14. R.W. Ogden. Non-linear Elastic Deformations. Dover Publication, New York, 1997.

15. Y.C. Fung. Biomechanics: Mechanical Properties of Living Tissues. New York: Springer, 1993.

16. W. Sun and M.S. Sacks. Finite element implementation of a generalized Fung-elastic constitutive model for planar soft tissues. Biomechanics and Modelling in Mechanobiology, 4:190-199, 2005.

17. G.A. Holzapfel, T.C. Gasser, and R.W. Ogden. A new constitutive framework for arterial wall mechanics and comparative study of material models. Journal of Elasticity, 61:1-48, 2000.

18. N. Promma, B. Raka, M. Grédiac, E. Toussaint, J.B. Le Cam, X. Balandraud, and F. Hild. Application of the virtual fields method to mechanical characterization of elastomeric materials. International Journal of Solids and Structures, 46:698-715, 2009. 